\title{
Protective efficacy by a novel multi-epitope vaccine, including MIC3, ROP8, and SAG1, against acute Toxoplasma gondii infection in BALB/c mice
}

\section{SamiraDodangeh ${ }^{\text {ae }}$ MahdiFasihi-Ramandi ${ }^{\mathrm{b}}$ AhmadDaryani ${ }^{\mathrm{c}}$ RezaValadan ${ }^{\mathrm{d}}$ HosseinAsgarian-}

Omran $^{\mathrm{d}}$ ZahraHosseininejad ${ }^{\mathrm{ce}}$ TooranNayeri,Chegeni ${ }^{\mathrm{ce}}$ AbdolSattarPagheh ${ }^{\mathrm{f}}$ JavadJavidnia $^{\mathrm{g}}$ Shahabe $\underline{\text { ddinSarvi }}^{\mathrm{c}}$

a

Department of Medical Parasitology and Mycology, Children Growth Research Center, Research Institute for Prevention of Non-Communicable Diseases, Qazvin University of Medical Sciences, Qazvin, Iran

b

Molecular Biology Research Center, Baqiyatallah University of Medical Sciences, Tehran, Iran c

Toxoplasmosis Research Center, Communicable Diseases Institute, Mazandaran University of Medical Sciences, Sari, Iran

d

Immunogenetics Research Center, School of Medicine, Mazandaran University of Medical Sciences, Sari, Iran

Student Research Committee, Mazandaran University of Medical Sciences, Sari, Iran f

Infectious Disease Research Center, Birjand University of Medical Sciences, Birjand, Iran $\mathrm{g}$

Department of Medical Mycology, Invasive Fungi Research Center, School of Medicine, Mazandaran University of Medical Sciences, Sari, Iran

${ }^{\mathrm{e}}$ Social Determinants of Health Research Center, Qazvin University of Medical Sciences, Qazvin, Iran

\section{Abstract}

Toxoplasma gondii is an intracellular apicomplexan parasite, which can cause a serious infectious disease in pregnant women and immunocompromised individuals. Therefore, the development of a polyvalent vaccine consisting of all stages of the parasite life cycle using the epitopes from tachyzoites, bradyzoites, and sporozoites is likely to be required for complete protective immunity. In this study, we designed protein vaccine candidate based on the prediction of specific epitopes (i.e., B cell and T cell) from three Toxoplasma gondii antigens. The MRS protein (MIC3: 30-180, ROP8: 85-185, and SAG1: 85-235) was expressed in Escherichia coli, and purification was performed using a HisTrap HP column and then we evaluated immunogenicity and protective property in BALB/c mice. Seventy-two mice were randomly divided into six groups, including three vaccinations (i.e., MRS, MRS-Freund, and MRS-Calcium Phosphate Nanoparticles (MRS- 
CaPNs)) and three control (i.e., Phosphate-buffered saline, Freund, and CaPNs) groups. All groups were immunized three times via subcutaneous injection within three-week intervals. In the vaccination groups, the BALB/c mice were injected with $20 \mu \mathrm{g}$ of MRS protein for the first time and $10 \mu \mathrm{g}$ of MRS for the next two times. Antibodies, cytokines, and splenocytes proliferation in the immunized mice were assayed using the enzyme-linked immunosorbent assay. Protective efficacy was analyzed by challenging the immunized mice with T. gondii of RH strain. Antibody, cytokine, and lymphocyte proliferation assays showed that the mice immunized with MRS induced stronger humoral and $\mathrm{T}$ helper type 1 cell-mediated immune responses, compared to the control mice. However, co-immunization with adjuvants (i.e., Freund and CaNPs) resulted in impaired immune responses. Effective protection against the parasite achieved an increase in survival time in the immunized mice, especially in the MRS-CaNPs group. The obtained results of the present study demonstrated that multi-epitope protein vaccination, MRS, is a potential strategy against toxoplasmosis infection. In addition, the vaccine co-delivered with CaPNs could provide an important key for vaccine candidate to control T. gondii infection.

Keywords

Multi-epitopeProtein vaccineToxoplasma gondiiFreundCalcium phosphate 